\title{
Attention-deficit hyperactivity disorder and anxiety disorders as precursors of bipolar disorder onset in adulthood
}

\author{
Sandra M. Meier, Barbara Pavlova, Søren Dalsgaard, Merete Nordentoft, Ole Mors, Preben B. Mortensen and \\ Rudolf Uher
}

\section{Background}

Attention-deficit hyperactivity disorder (ADHD) and anxiety disorders have been proposed as precursors of bipolar disorder, but their joint and relative roles in the development of bipolar disorder are unknown.

\section{Aims}

To test the prospective relationship of ADHD and anxiety with onset of bipolar disorder.

\section{Method}

We examined the relationship between ADHD, anxiety disorders and bipolar disorder in a birth cohort of 2409236 individuals born in Denmark between 1955 and 1991. Individuals were followed from their sixteenth birthday or from January 1995 to their first clinical contact for bipolar disorder or until December 2012. We calculated incidence rates per 10000 person-years and tested the effects of prior diagnoses on the risk of bipolar disorder in survival models.

\section{Results}

Over 37394865 person-years follow-up, 9250 onsets of bipolar disorder occurred. The incidence rate of bipolar disorder was $2.17(95 \% \mathrm{Cl}$ 2.12-2.19) in individuals with no prior diagnosis of
ADHD or anxiety, 23.86 (95\% Cl 19.98-27.75) in individuals with a prior diagnosis of ADHD only, $26.05(95 \% \mathrm{Cl} 24.47-27.62)$ in individuals with a prior diagnosis of anxiety only and 66.16 $(95 \% \mathrm{Cl} 44.83-87.47)$ in those with prior diagnoses of both ADHD and anxiety. The combination of ADHD and anxiety increased the risk of bipolar disorder 30-fold (95\% Cl 21.66-41.40) compared with those with no prior ADHD or anxiety.

\section{Conclusions}

Early manifestations of both internalising and externalising psychopathology indicate liability to bipolar disorder. The combination of ADHD and anxiety is associated with a very high risk of bipolar disorder.

\section{Declaration of interest}

None.

\section{Keywords}

Bipolar disorder; anxiety disorders; attention-deficit/hyperactivity disorder; developmental psychopathology; early identification of risk; internalizing disorders; externalising disorders.

\section{Copyright and usage}

(c) The Royal College of Psychiatrists 2018

\section{Rationale for predicting bipolar disorder}

Prediction of bipolar disorder is important from clinical and public health perspectives, because bipolar disorder is associated with a high risk of suicide and other adverse outcomes, is often initially misdiagnosed as depression and requires specific treatment different from that for major depressive disorder. ${ }^{1}$ In addition, understanding the developmental psychopathology leading to bipolar disorder and the early identification of individuals at risk are essential for the development of early pre-emptive interventions that may prevent the onset of bipolar disorder. ${ }^{2,3}$

\section{Internalising and externalising psychopathology as antecedent to bipolar disorder}

One way of indexing early liability to bipolar disorder is as a combination of externalising and internalising domains of psychopathology. Bipolar disorder manifests with both internalising (depression, anxiety) and externalising (impulsivity, risk-taking) symptoms. It has been proposed that a 'dysregulation' profile characterised by increases in both externalising (for example inattention and hyperactivity) and internalising (for example anxiety) psychopathology may identify youth at high risk for developing bipolar disorder. ${ }^{4}$ The evidence for an association between the dysregulation profile and liability for bipolar disorder has been mixed. Although the dysregulation profile was found in children diagnosed with broadly defined paediatric bipolar disorder ${ }^{5}$ and in offspring of parents with bipolar disorder, ${ }^{6}$ longitudinal studies report inconsistent evidence for an association between a dysregulation profile early in life and bipolar disorder onset in adulthood. ${ }^{7,8}$ At the level of diagnosed disorders, both internalising (for example anxiety disorders) and externalising (for example attention-deficit hyperactivity disorder (ADHD)) disorders with childhood onset have been proposed to index early manifestations of liability for bipolar disorder.

\section{Anxiety disorders and ADHD as precursors of bipolar disorder}

Childhood-onset anxiety disorders are associated with a family history of bipolar disorder ${ }^{2,9,10}$ and have been reported to precede and predict bipolar disorder in familial high-risks studies. ${ }^{10}$ The evidence for ADHD being associated with liability for bipolar disorder is less consistent: ADHD is more common among offspring of parents with bipolar disorder ${ }^{9}$ and bipolar disorder is more common in relatives of individuals with ADHD, ${ }^{11}$ but ADHD did not consistently predict bipolar disorder in longitudinal familial high-risk studies. ${ }^{12}$ At present, it is unknown whether the combination of ADHD and anxiety disorders increases the risk of bipolar disorder more than either disorder alone.

\section{Research questions}

Prior evidence on whether ADHD and anxiety alone or in combination predict bipolar disorder may be inconsistent because it is limited to familial high-risk samples of moderate size, where the number of prospectively ascertained onsets may not be sufficient to conduct tests with adequate statistical power and existing genetic diathesis may confound the apparent associations. 
Additional inconsistencies may have been introduced by mixing of narrowly defined bipolar disorder that typically onsets in late adolescence or adulthood with broader definitions of paediatric bipolar disorder. ${ }^{5,8,12,13}$ Therefore, we aim to test the prospective relationship of prior ADHD and anxiety with onset of bipolar disorder in adulthood in a large representative population sample. Specifically, we tested the hypotheses that (a) prior ADHD predicts adult-onset bipolar disorder; (b) prior anxiety disorders predict adult-onset bipolar disorder; (c) the combination of prior ADHD and prior anxiety diagnoses predicts bipolar disorder more strongly than either disorder alone.

\section{Method}

\section{Participants}

We conducted a nationwide follow-up study using linked data from the Danish Psychiatric Central Research Register, ${ }^{14}$ The Danish National Patient Register ${ }^{15}$ and the Danish Civil Registration System. ${ }^{16}$ Data were collected on individuals born in Denmark between 1 May 1955 and 31 December 1991. The follow-up-time started on their sixteenth birthday or on 1 January 1995, whichever came later, to ensure follow-up to a minimum age of 21 years. Follow-up ended at date of emigration from Denmark, date of death or 31 December 2012, whichever came first. This provided a minimum follow-up period of 5 years between the ages of 16 to 57 years. The cohort was restricted to individuals with known parents resulting in a total of 2409236 individuals. A detailed description of the cohort is available in Supplementary Table 1 available at https://doi.org/10.1192/bjp.2018.111.

The present study was completed as part of the iPSYCH project. ${ }^{17}$ The procedures have been reviewed and approved by the Scientific Ethics Committee in the Central Denmark Region, which has concluded that the study does not involve risk to participants health or other risk and has approved the use of data for the above analyses without the need to collect informed consent from individual participants. The project has also been approved by the Danish Health Data Authority and the Danish Data Protection Agency.

\section{Data sources}

Data on cohort members were obtained by linking three Danish population-based registers. All live-born children and new residents in Denmark since 1968 are assigned a unique personal identification number, which is used across all registration systems enabling perfect linkage. The Danish Civil Registration System contains information on sex, date and place of birth, vital status (continuously updated) and parents' personal identifiers. ${ }^{16}$ The Danish National Hospital Registry has recorded all in-patient treatments in Danish hospitals since 1977. This registry has also recorded all out-patient and emergency room contacts from 1995 onward. ${ }^{15}$ The Danish Psychiatric Central Research Register covers all psychiatric in-patient facilities since 1969 in addition to out-patient contacts since $1995 .{ }^{14}$ For both registers, diagnostic information was based on ICD-8 from 1977 to 1993 and on ICD-10 from 1994 onward. ${ }^{18,19}$ The registered admissions are representative of the entire Danish population because every individual living in Denmark has equal and free access to both somatic and mental healthcare.

\section{Predictors}

The main predictor variables were defined as a clinical contact for ADHD or an anxiety disorder (generalised anxiety disorder, panic disorder, agoraphobia, social anxiety disorder, specific phobia, obsessive-compulsive disorder, post-traumatic stress disorder) at any time from early childhood until the follow-up point. We selected $\mathrm{ADHD}$ as a representative externalising disorder because it is the childhood disorder that provides most information about the externalising psychopathology spectrum and because it is primarily defined by externalising symptoms. ${ }^{20}$ This contrasts with disruptive disorders, such as oppositional-defiant disorder, which are defined by a combination of internalising and externalising symptoms and show developmental continuities with early temperament emotionality and later emotional disorders. ${ }^{21-23}$ We identified all patients diagnosed with ADHD or anxiety disorders using definitions consistent with previous studies, which have established the validity of registry diagnoses of $\mathrm{ADHD}^{24,25}$ and anxiety disorders. ${ }^{26}$ During the follow-up period, the Danish Psychiatric Central Research Register and the Danish National Patient Register consistently used the ICD-10. ${ }^{19}$ However, prior to January 1994, these registries used the ICD-8. ${ }^{18}$ In order to maximise the capture of childhood-onset diagnoses across a large cohort, we used prior diagnoses of ADHD and anxiety disorders recorded with either ICD-10 or ICD- 8 in the primary analyses. Specifically, we identified all patients diagnosed with ADHD (ICD-10: F90; ICD-8: 308.01) and anxiety disorders (ICD-10: F40.00-F41.19, F42.00-F43.10; ICD-8: 300.09, 300.29, 300.39), as described in prior studies. ${ }^{24-26}$ The date of onset was defined as the day of the first recorded clinical contact for the diagnosis in question. Since the diagnostic conceptualisation of ADHD and, to a lesser extent anxiety, changed from the ICD-8 to ICD-10, we also completed sensitivity analyses restricted to diagnoses of ADHD and anxiety disorders recorded with the ICD-10 between January 1994 and December 2012.

\section{Outcome}

The outcome variable was defined as the first recorded clinical contact with the diagnosis of a manic episode, hypomanic episode, mixed affective episode or bipolar disorder (ICD-10: F30F31, F38.00).

\section{Covariates}

Information on age (16-20, 21-25, 26-30, 31-35, 36-40, 41-45, 46$50,>50$ years), gender, calendar period (1955-1965, 1966-1975, 1976-1985, <1985), maternal and paternal age at time of birth $(\leq 20,21-25,26-30,31-35,<35)$ were derived from the Danish Civil Registration System.

\section{Censoring}

We censored individuals with a diagnosis of autism spectrum disorders because it is not compatible with reliable diagnosis of bipolar disorder. Information on autism spectrum disorders (ICD-8: 299.00-299.03; ICD-10: F84.00-F84.12; F84.50-F84.99) was retrieved from the Danish Psychiatric Central Research Register and the Danish National Patient Register.

\section{Data analysis}

The data were examined by survival analysis. Cohort members were followed from their sixteenth birthday or 1 January 1995 until the onset of bipolar disorder, date of death, date of emigration from Denmark, or 31 December 2012, whichever occurred first. The survival time was initiated at age 16 (rather than at the onset of ADHD or anxiety) so that the risk for adult-onset bipolar disorder associated with early- and later-onset precursors is assessed in a comparable fashion.

In incidence analyses, we first determined the risks of bipolar disorder in patients with ADHD or anxiety disorders compared 
with the general population, using a log-linear Poisson regression model as implemented in SAS, version 9.4 and adjusted for calendar year, age, gender, parental age at time of birth, and the interaction of age with gender. Age, calendar year and the occurrence of an ADHD or anxiety disorder diagnosis were treated as time dependent variables, whereas all other variables were considered time independent. Analyses with additional adjustment for family history of mental disorder in parents are provided in Supplementary Table 2.

To confirm that the results were not unduly influenced by changes in diagnostic classification, we repeated the main tests in sensitivity analyses with a sample restricted to individuals where all disorders were diagnosed according to ICD-10. All sensitivity analyses were adjusted for calendar year, age, gender, parental age at time of birth, and the interaction of age with gender. Next, following the recommendation that additive risk models are most informative for both population health implications and causal mechanisms, ${ }^{27}$ we tested whether the risk of bipolar disorder in individuals with prior diagnoses of both ADHD and anxiety differs from the addition of risks conferred by each precursor in isolation, using additive hazards models with the Aalen function through the R package 'timereg. ${ }^{28}$ Additional analyses tested the specificity of predictors and outcomes (see Supplementary Tables 2, 3 and 4).

\section{Results}

\section{Risk of bipolar disorder}

From 1 January 1995 to 31 December 2012 a total of 2409236 individuals were included in the study cohort. In 115571 (4.8\%) cohort members, follow-up was ended before the end of the study, because 43775 died, 67586 emigrated from Denmark, 1232 were lost to follow-up and 2978 were censored because of an autism diagnosis. Over the remaining 37394865 person-years of follow-up, 9250 individuals with bipolar disorder were identified, with a mean age at onset of 33.49 years (s.d. $=9.84$ ). The overall incidence rate of bipolar disorder among individuals aged $16-57$ years was 2.47 per
10000 person-years (95\% CI 2.42-2.52). Among individuals with no prior diagnosis of ADHD or anxiety, the incidence rate of bipolar disorder was 2.17 (95\% CI 2.12-2.19; Fig. 1).

\section{Prior ADHD and risk of bipolar disorder}

There were 13628 individuals with a prior diagnosis of ADHD. During 66353 person-years of follow-up of these individuals, 182 new onsets of bipolar disorder were recorded, resulting in an incidence rate of 27.43 per 10000 person-years (95\% CI 23.44-31.41). After excluding individuals with prior anxiety disorders, the incidence rate of bipolar disorder in those with prior ADHD only was 23.86 per 10000 person-years (95\% CI 19.98-27.75; Fig. 1). A prior diagnosis of ADHD increased the incidence of bipolar disorder 11.29-fold (95\% CI 9.72-13.03; $P<0.001$; Table 1) compared with individuals without ADHD. Adjustment for gender, age, interaction of gender and age, calendar year and parental age reduced the incidence rate ratio slightly to 10.00 (95\% CI 8.58-11.57; $P<0.001)$. In sensitivity analysis restricted to individuals diagnosed according to ICD-10, 168 new cases of bipolar disorder were recorded among individuals with ICD-10 diagnosis of ADHD resulting in an incidence rate ratio of 11.72 (95\% CI 9.99-13.66; $P<0.001)$.

\section{Prior anxiety disorders and risk of bipolar disorder}

There were 56349 individuals diagnosed with one or more anxiety disorders. During 408358 person-years of follow-up of these individuals, 1086 new cases of bipolar disorder were recorded, resulting in an incidence rate of 26.59 per 10000 person-years (95\% CI 25.0128.18; Fig. 1). After excluding individuals with prior $\mathrm{ADHD}$, the incidence rate of bipolar disorder in those with prior anxiety only was 26.05 per 10000 person-years (95\% CI 24.47-27.62; Fig. 1). Overall, prior diagnosis of anxiety disorder increased the incidence of bipolar disorder 12.05-fold (95\% CI 11.30-12.83; $P<0.001$; Table 1) compared with individuals without a prior diagnosis of an anxiety disorder. Adjustment for gender, age, interaction of gender and age, calendar year and parental age reduced the incidence rate ratio slightly to 9.61 (95\% CI 9.01-10.24; $P<0.001)$. In

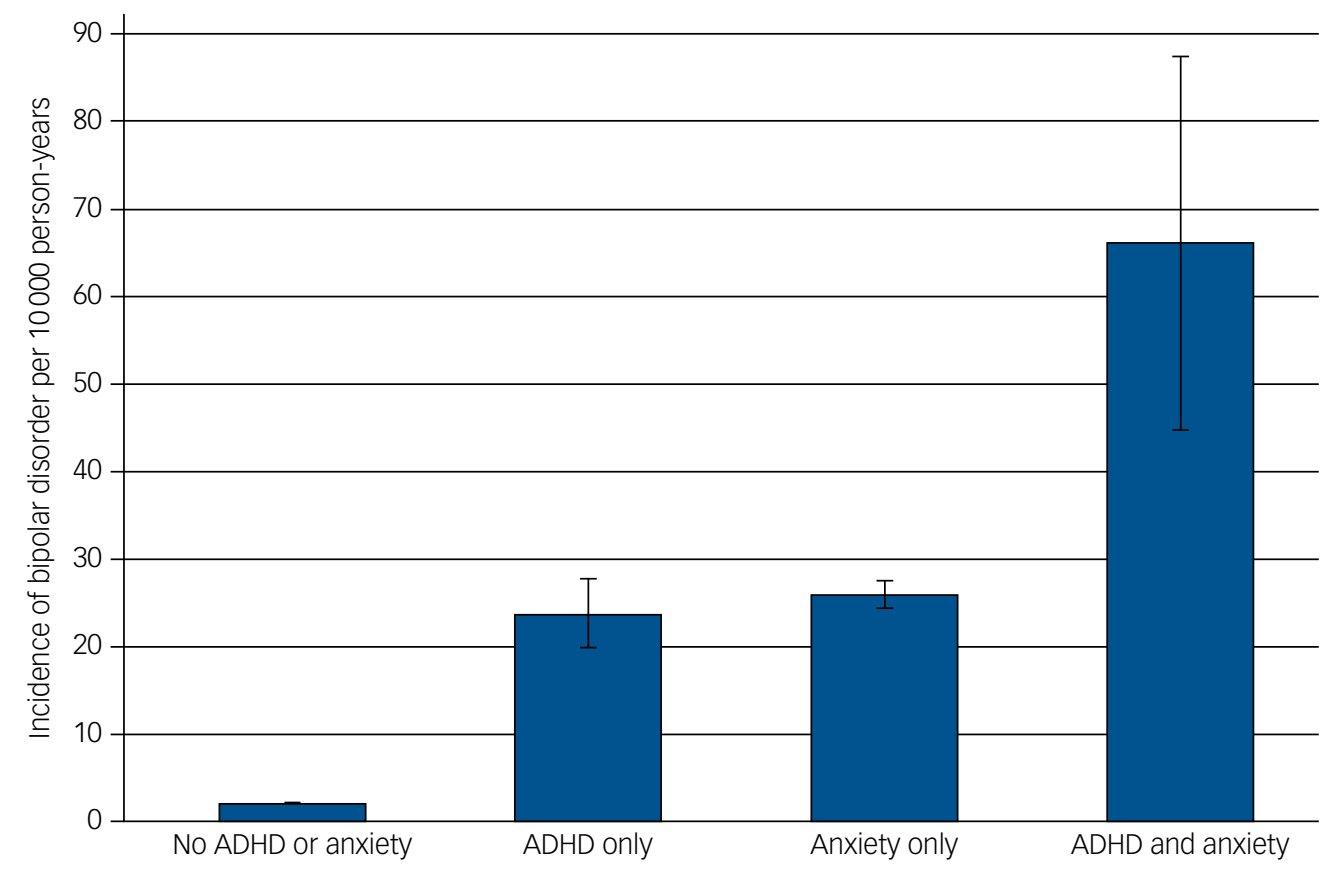

Fig. 1 Incidence rates of bipolar disorder in adults with and without prior diagnoses of attention-deficit hyperactivity disorder (ADHD) and/or anxiety disorders. Error bars indicate $95 \%$ Cls. 
Table 1 Risk of bipolar disorder associated with prior attention-deficit hyperactivity disorder (ADHD) and anxiety diagnoses (1995-2012)

\begin{tabular}{|c|c|c|c|c|c|}
\hline & \multirow[t]{3}{*}{ Onset of bipolar disorder, $n$} & \multicolumn{4}{|c|}{ Incidence rate ratio (IRR) } \\
\hline & & \multicolumn{2}{|c|}{ Crude } & \multicolumn{2}{|c|}{ Adjusted $^{a}$} \\
\hline & & IRR & $95 \% \mathrm{Cl}$ & IRR & $95 \% \mathrm{Cl}$ \\
\hline \multicolumn{6}{|l|}{ Single diagnosis effects } \\
\hline ADHD & 182 & 11.29 & $9.72-13.03$ & 10.00 & $8.58-11.57$ \\
\hline Anxiety disorders & 1086 & 12.05 & $11.30-12.83$ & 9.61 & $9.01-10.24$ \\
\hline \multicolumn{6}{|l|}{ Joint effects } \\
\hline No ADHD or anxiety disorder & 8019 & 1.00 & Reference & 1.00 & Reference \\
\hline ADHD only & 145 & 10.99 & $9.28-12.90$ & 10.13 & $8.54-11.92$ \\
\hline Anxiety disorders only & 1049 & 11.99 & $11.24-12.78$ & 9.61 & $9.00-10.25$ \\
\hline ADHD and anxiety disorders & 37 & 30.46 & $21.66-41.40$ & 21.15 & $15.02-28.78$ \\
\hline
\end{tabular}

sensitivity analysis restricted to individuals where both anxiety and bipolar disorders were diagnosed according to ICD-10, there were 1014 new cases of bipolar disorder among individuals with a prior ICD-10 diagnosis of anxiety disorder, resulting in an incidence rate ratio of 9.70 (95\% CI 9.07-10.36; $P<0.001)$.

\section{ADHD, anxiety disorders and risk of bipolar disorder}

There were 2105 individuals with prior diagnoses of both ADHD and anxiety disorders. During 5593 person-years of follow-up of these individuals, 37 new onsets of bipolar disorder occurred, resulting in an incidence rate of 66.16 per 10000 person-years (95\% CI 44.84-87.47; Fig. 1). The combination of prior diagnoses of ADHD and anxiety disorders increased the risk of bipolar disorder 30.46-fold (95\% CI 21.66-41.40; $P<0.001$; Table 1) compared with those with no prior diagnosis of anxiety or ADHD. After adjustment for gender, age, interaction of gender and age, calendar year and paternal age, the incidence rate ratio was 21.15 (95\% CI $15.02-$ 28.78; $P<0.001)$. The risk conferred by a combination of ADHD and anxiety was substantially higher than the risk conferred by either ADHD or anxiety alone. The test of interaction between $\mathrm{ADHD}$ and anxiety did not show statistically significant departure from additive risk $(P=0.103)$, suggesting that the risk in those with both prior disorders approximately corresponds to the addition of risks associated with prior ADHD and prior anxiety. In sensitivity analysis restricted to individuals where ADHD, anxiety and bipolar disorders were diagnosed according to ICD-10, the combination of prior ICD-10 diagnoses of ADHD and anxiety disorder resulted in a 22 -fold increased risk of bipolar disorder (incidence rate ratio $22.35,95 \% \mathrm{CI} 15.79-30.54 ; P<0.001$ ). Additional analyses probed the specificity of associations to $\mathrm{ADHD}$, anxiety disorder and bipolar disorder (Supplementary Tables 2, 3 and 4).

\section{Discussion}

\section{Main findings}

In a representative national register, we found that prior diagnoses of ADHD and anxiety disorders were associated with similarly increased risk of bipolar disorder. The risk of bipolar disorder increased further in those who received prior diagnoses of both ADHD and anxiety. The results of sensitivity analyses suggested that the findings are robust and were not influenced by changes in mental disorder definitions from ICD-8 to ICD-10. These findings suggest that manifestations of both internalising and externalising psychopathology indicate increased risk of bipolar disorder.

The effect sizes of the associations were substantial: ADHD or anxiety increased the risk of adult-onset bipolar disorder 10-fold and the combination of ADHD and anxiety increased the risk of adult-onset bipolar disorder 30-fold, compared with those with no prior diagnosis of either ADHD or anxiety. Therefore, the present findings have implications for further research on the aetiology of bipolar disorder and for public health.

We selected ADHD and anxiety as prototypes of externalising and internalising disorders that do not share diagnostic symptoms with bipolar disorder. Unlike oppositional-defiant disorder that has both internalising and externalising components, ${ }^{21-23}$ ADHD is a relatively pure externalising disorder that is tightly linked with the externalising psychopathology spectrum. ${ }^{20}$ Anxiety disorders frequently co-occur with bipolar disorder, ${ }^{29}$ but have distinct defining symptoms and clinical course ${ }^{30}$ and are the prototype of internalising psychopathology. ${ }^{31,32}$ The strong prospective heterotypic associations between both ADHD and anxiety and bipolar disorder suggest that the diathesis for bipolar disorder overlaps with both externalising and internalising psychopathology spectra. This is consistent with the previously proposed dysregulation profile, ${ }^{8}$ the extensive overlaps between genetic risk variants associated with a range of childhood- and adult-onset disorders ${ }^{33,34}$ and with the disproportionately frequent comorbidity of bipolar disorder with anxiety ${ }^{29,30}$ and with ADHD. ${ }^{35}$ Prospective studies aiming to examine the developmental precursors of bipolar disorder should assess and consider both internalising and externalising psychopathology as potential antecedents. Aetiological research may benefit from a transdiagnostic perspective where bipolar disorder is examined as one of the endpoints of a broad vulnerability to psychopathology.

\section{Implications for clinical practice}

Quantification of risk for bipolar disorder in each subgroup is essential to inform public health and clinical decisions. The present registry-based data suggest that a substantial proportion of bipolar disorder onsets are concentrated in a small section of the population. The 10-fold increase in risk of bipolar disorder among individuals with a history of ADHD or anxiety may indicate increased caution, especially after exposure to additional factors that are associated with onset of bipolar disorder. The 30-fold increase in risk of bipolar disorder among those with a history of both ADHD and anxiety may indicate the need for targeted early pre-emptive interventions. The finding of our supplementary analyses (that individuals with a history of both ADHD and anxiety are also at markedly increased risk for schizophrenia in addition to bipolar disorder underline the importance of this group as a potential target for prevention (Supplementary Table 4).

\section{Strengths and limitations}

The present study benefits from a large representative nationwide sample, which enables highly generalisable results that are free of 
biases associated with research that enrols individual participants. The results were robust and not unduly influenced by changes in the diagnostic classification system. Yet, our results should be interpreted with two limitations in mind. First, we identified diagnoses of bipolar disorder, ADHD and anxiety from a register that covers in-patient and specialist out-patient care, but not care received through general practitioners. The absence of records of diagnoses from general practitioners may have resulted in an underestimation of the prevalence of mental disorders, in particular ADHD and anxiety disorders. Additionally, separation anxiety disorder is not adequately covered in the registry and could not be included. ${ }^{26}$ It is also likely that the recorded diagnoses reflect individuals with the more severe cases that tend to be seen in specialist services. Thus, our results may not generalise to milder forms of ADHD and anxiety disorders. However, the sample represents individuals who access services and, therefore, provide opportunities for interventions. It is also possible that individuals with multiple comorbid disorders may be more likely to access secondary care than those with a single disorder. Such differences in help-seeking may have affected the relative proportions of individuals with single disorders $v$. comorbid ADHD and anxiety in our analyses. However, since the vast majority of individuals with bipolar disorder in Denmark receive treatment through secondary care, ${ }^{36}$ help-seeking behaviour should not have a major impact on the reported relationships with bipolar disorder.

A second limitation is that treatment contact is delayed in most affected individuals. Individuals not yet diagnosed with bipolar disorder might have had early manifestations of a mood disorder that were misclassified as a different diagnosis. We have sought to minimise the risk of misclassification bias by selecting antecedent diagnoses that do not have direct symptom overlap with bipolar disorder and by focusing on onsets of bipolar disorder occurring in late adolescence and adulthood. Therefore, the enhanced risk is unlikely to be because of coexisting untreated bipolar disorder alone.

In conclusion, we have observed that prior diagnoses of ADHD and anxiety disorders are associated with an increased risk of bipolar disorder. The very high risk of bipolar disorder among individuals with a history of both ADHD and anxiety supports the hypothesis that the propensity to bipolar disorder overlaps with both internalising and externalising psychopathology. Individuals with both ADHD and anxiety disorders may be candidates for pre-emptive interventions to reduce the risk of bipolar disorder.

Sandra M. Meier, PhD, Postdoctoral Fellow, Child and Adolescent Mental Health Centre-Mental Health Services Capital Region, Copenhagen Region, Psychosis Research Unit, Aarhus University Hospital and The Lundbeck Foundation Initiative for Integrative Psychiatric Research, iPSYCH, Denmark; Barbara Pavlova, PhD, Assistant Professor, Department of Psychiatry, Dalhousie University and Nova Scotia Health Authority, Canada; Søren Dalsgaard, MD, The Lundbeck Foundation Initiative for Integrative Canada; Søren Dalsgaard, MD, The Lundbeck Foundation Initiative for Integrative
Psychiatric Research, iPSYCH, National Centre for Register-Based Research, NCRR, Psychiatric Research, iPSYCH, National Centre for Register-Based Research, NCRR,
Aarhus University, Denmark and Department for Child and Adolescent Psychiatry, Hospital of Telemark, Norway; Merete Nordentoft, Dr Med Sc, Professor, The Lundbeck Foundation Initiative for Integrative Psychiatric Research, IPSYCH, and Copenhagen University Hospital, Mental Health Center Copenhagen, Denmark; Ole Mors, MD, Professor, Psychosis Research Unit, Aarhus University Hospital and The Lundbeck Foundation Intiative for Integrative PSychiatric Research, PSYCH, Denmark Preben B. Mortensen, Dr Med SC, Professor, The Lundbeck Foundation Initiative for Integrative B. Mortensen, Dr Med SC, Professor, The Lundbeck Foundation Initiative for Integrative
Psychiatric Research, IPSYCH, National Centre for Register-Based Research, NCRR, Aarhus University and CIRRAU - Centre for Integrated Register-based Research, Aarhus University; Rudolf Uher, MD, Professor, Department of Psychiatry, Dalhousie University and Senior Researcher, Nova Scotia Health Authority, Canada

Correspondence: Rudolf Uher, Dalhousie University, 5909 Veterans Memorial Drive, Halifax, NS, B3H 2E2, NS, Canada. Email: uher@dal.ca

First received 1 Jan 2018, final revision 7 May 2018, accepted 7 May 2018

\section{Supplementary material}

Supplementary material is available online at https://doi.org/10.1192/bjp.2018.111.

\section{Funding}

This study was funded by the Lundbeck Foundation, Denmark (Grant number R155-2014-1724). S.M.M. has been supported by the Mental Health Services Capital Region Copenhagen
Denmark. B.P. has been supported by the Nova Scotia Health Research Foundation. S.D. has received funding from the European Commission as part of the Comorbid Conditions of Attention-deficit/hyperactivity disorder (COCA) project. R.U.'s contribution has been supported by funding from the Canada Research Chairs Program (award number 231397), the Canadian Institutes of Health Research (Grant reference numbers 124976, 142738 and 148394), Nova Scotia Health Research Foundation (275319, 1716 and 353892) and the Dalhousie Medical Research Foundation. The funders had no role in study design, data collection, data analysis, interpretation of results, writing of the manuscript or the decision to submit the manuscript for publication.

\section{Acknowledgements}

We thank the iPSYCH team (http://ipsych.au.dk/) for support.

\section{References}

1 Grande I, Berk M, Birmaher B, Vieta E. Bipolar disorder. Lancet 2016; 387: 1561-72.

2 Nurnberger Jr Jl, McInnis M, Reich W, Kastelic E, Wilcox HC, Glowinski A, et al. A high-risk study of bipolar disorder. Childhood clinical phenotypes as precursors of major mood disorders. Arch Gen Psychiatry 2011; 68: 1012-20.

3 Uher R, Cumby J, MacKenzie LE, Morash-Conway J, Glover JM, Aylott A, et al. A familial risk enriched cohort as a platform for testing early interventions to prevent severe mental illness. BMC Psychiatry 2014; 14: 344

4 Mick E, Biederman J, Pandina G, Faraone SV. A preliminary meta-analysis of the child behavior checklist in pediatric bipolar disorder. Biol Psychiatry 2003; 53: 1021-27.

5 Faraone SV, Althoff RR, Hudziak JJ, Monuteaux M, Biederman J. The CBCL predicts DSM bipolar disorder in children: a receiver operating characteristic curve analysis. Bipolar Disord 2005; 7: 518-24.

6 Maoz H, Goldstein T, Axelson DA, Goldstein BI, Fan J, Hickey MB, et al. Dimensional psychopathology in preschool offspring of parents with bipolar disorder. J Child Psychol Psychiatry 2014; 55: 144-53.

7 Biederman J, Petty CR, Monuteaux MC, Evans M, Parcell T, Faraone SV, et al. The Child Behavior Checklist-Pediatric Bipolar Disorder profile predicts a subsequent diagnosis of bipolar disorder and associated impairments in ADHD youth growing up: a longitudinal analysis. J Clin Psychiatry 2009; 70: 732-40.

8 Althoff RR, Verhulst FC, Rettew DC, Hudziak JJ, van der EJ. Adult outcomes of childhood dysregulation: a 14-year follow-up study. J Am Acad Child Adolesc Psychiatry 2010; 49: 1105-16.

9 Rasic D, Hajek T, Alda M, Uher R. Risk of mental illness in offspring of parents with schizophrenia, bipolar disorder, and major depressive disorder: a metaanalysis of family high-risk studies. Schizophr Bull 2014; 40: 28-38.

10 Duffy A, Alda M, Hajek T, Sherry SB, Grof P. Early stages in the development of bipolar disorder. J Affect Disord 2010; 121: 127-35.

11 Larsson H, Ryden E, Boman M, Langstrom N, Lichtenstein P, Landen M. Risk of bipolar disorder and schizophrenia in relatives of people with attention-deficit hyperactivity disorder. Br J Psychiatry 2013; 203: 103-6.

12 Duffy $A$. The nature of the association between childhood ADHD and the development of bipolar disorder: a review of prospective high-risk studies. Am J Psychiatry 2012; 169: 1247-55.

13 Diler RS, Birmaher B, Axelson D, Goldstein B, Gill M, Strober M, et al. The Child Behavior Checklist (CBCL) and the CBCL-bipolar phenotype are not useful in diagnosing pediatric bipolar disorder. J Child Adolesc Psychopharmacol 2009; 19: $23-30$.

14 Mors O, Perto GP, Mortensen PB. The Danish Psychiatric Central Research Register. Scand J Public Health 2011; 39: 54-7.

15 Lynge E, Sandegaard JL, Rebolj M. The Danish National Patient Register. Scand J Public Health 2011; 39: 30-3.

16 Pedersen CB. The Danish Civil Registration System. Scand J Public Health 2011; 39: $22-5$.

17 iPSYCH. The Lunbeck Foundation Initiative for Integrative Psychiatric Research iPSYCH, 2017 (http://ipsych.au.dk/).

18 World Health Organization. International Statistical Classification of Diseases and Related Health Problems (ICD-8). WHO, 1967.

19 World Health Organization. The ICD-10 Classification of Mental and Behavioural Disorders: Clinical Descriptions and Diagnostic Guidelines. WHO, 1992.

20 Carragher N, Krueger RF, Eaton NR, Markon KE, Keyes KM, Blanco C, et al. ADHD and the externalizing spectrum: direct comparison of categorical, continuous, and hybrid models of liability in a nationally representative sample. SoC Psychiatry Psychiatr Epidemiol 2014; 49: 1307-17. 
21 Stringaris A, Maughan B, Goodman R. What's in a disruptive disorder? Temperamental antecedents of oppositional defiant disorder: findings from the Avon longitudinal study. J Am Acad Child Adolesc Psychiatry 2010; 49: 474-83.

22 Krieger FV, Polanczyk VG, Goodman R, Rohde LA, Graeff-Martins AS, Salum G, et al. Dimensions of oppositionality in a Brazilian community sample: testing the DSM-5 proposal and etiological links. J Am Acad Child Adolesc Psychiatry 2013; 52: 389-400.

23 Whelan YM, Stringaris A, Maughan B, Barker ED. Developmental continuity of oppositional defiant disorder subdimensions at ages 8,10 , and 13 years and their distinct psychiatric outcomes at age 16 years. J Am Acad Child Adolesc Psychiatry 2013; 52: 961-9.

24 Dalsgaard S, Ostergaard SD, Leckman JF, Mortensen PB, Pedersen MG. Mortality in children, adolescents, and adults with attention deficit hyperactivity disorder: a nationwide cohort study. Lancet 2015; 385: 2190-6.

25 Linnet KM, Wisborg K, Secher NJ, Thomsen PH, Obel C, Dalsgaard S, et al. Coffee consumption during pregnancy and the risk of hyperkinetic disorder and ADHD: a prospective cohort study. Acta Paediatr 2009; 98: 173-9.

26 Meier SM, Petersen L, Mattheisen M, Mors O, Mortensen PB, Laursen TM. Secondary depression in severe anxiety disorders: a population-based cohort study in Denmark. Lancet Psychiatry 2015; 2: 515-23.

27 Rothman KJ, Greenland S, Lash TL. Modern Epidemiology. Wolter Kluwe Health, 2008

28 Rod NH, Lange T, Andersen I, Marott JL, Diderichsen F. Additive interaction in survival analysis: use of the additive hazards model. Epidemiology 2012; 23: 733-7.

29 Pavlova B, Perlis RH, Alda M, Uher R. Lifetime prevalence of anxiety disorders in people with bipolar disorder: a systematic review and meta-analysis. Lancet Psychiatry 2015; 2: 710-7.
30 Pavlova B, Perlis RH, Mantere O, Sellgren CM, Isometsa E, Mitchell PB, et al. Prevalence of current anxiety disorders in people with bipolar disorder during euthymia: a meta-analysis. Psychol Med 2017; 47: 1107-15.

31 Eaton NR, Krueger RF, Markon KE, Keyes KM, Skodol AE, Wall M, et al. The structure and predictive validity of the internalizing disorders. J Abnorm Psychol 2013; 122: 86-92.

32 Olino TM, Dougherty LR, Bufferd SJ, Carlson GA, Klein DN. Testing models of psychopathology in preschool-aged children using a structured interviewbased assessment. J Abnorm Child Psychol 2014; 42: 1201-11.

33 Cross-Disorder Group of the Psychiatric Genomics Consortium. Identification of risk loci with shared effects on five major psychiatric disorders: a genomewide analysis. Lancet 2013; 381: 1371-9.

34 van Hulzen KJ, Scholz CJ, Franke B, Ripke S, Klein M, McQuillin A, et al. Genetic overlap between attention-deficit/hyperactivity disorder and bipolar disorder: evidence from genome-wide association study meta-analysis. Biol Psychiatry 2017: 82: 634-41.

35 Wang HR, Jung YE, Chung SK, Hong J, Ri KN, Kim MD, et al. Prevalence and correlates of bipolar spectrum disorder comorbid with ADHD features in nonclinical young adults. J Affect Disord 2017; 207: 175-80.

36 Kessing LV, Hansen HV, Hvenegaard A, Christensen EM, Dam H, Gluud C, et al. Treatment in a specialised out-patient mood disorder clinic v. standard outpatient treatment in the early course of bipolar disorder: randomised clinical trial. Br J Psychiatry 2013; 202: 212-9. 